\title{
Carrier mobility in a polar semiconductor measured by an optical pump-probe technique
}

\author{
Muneaki Hase ${ }^{a}$ \\ Institute of Applied Physics, University of Tsukuba, 1-1-1 Tennodai, Tsukuba 305-8573, Japan and PRESTO, \\ Japan Science and Technology Agency, 4-1-8 Honcho, Kawaguchi, Saitama 332-0012, Japan
}

(Received 11 December 2008; accepted 27 February 2009; published online 19 March 2009)

\begin{abstract}
Ultrafast dephasing of the plasmonlike longitudinal optical phonon-plasmon coupled (LOPC) mode in highly doped $n$-GaAs has been investigated by using a femtosecond optical pump-probe technique with $40 \mathrm{THz}$ bandwidth as a function of photodoping levels. The direct measurement of plasmon damping with the help of a wavelet analysis enables us to extract carrier (electron) mobility, which decreases with increasing the photodoping levels. It is found that the mobility is suppressed at high photodoping levels due to electron-hole scattering, while it is enhanced near a critical density, being plausibly attributed to the strong coherent coupling of the LO phonon with the plasmon. (c) 2009 American Institute of Physics. [DOI: 10.1063/1.3103275]
\end{abstract}

Carrier mobility is one of the most important properties in semiconductor devices, such as high electron mobility transistor and field-effect transistor, both of which are capable for realizing terahertz $\left(10^{12} \mathrm{~Hz}\right)$ ultrahigh-speed operation in telecommunication and optical memory. The carrier mobility is determined by $\mu=e \tau / m^{*}$, where $e$ denotes electron charge, $\tau$ is the carrier relaxation time introduced in the relaxation time approximation, ${ }^{1}$ and $m^{*}$ represents electron effective mass in $n$-type semiconductors. Here, the carrier relaxation time $\tau$ includes all scattering processes, such as electron-hole scattering, electron-phonon scattering (Fröhlich and deformation potentials), and scattering by defects and disorders. In general, the time scale of $\tau$ is less than a few hundred femtoseconds. While the carrier mobility has recently been obtained by using terahertz time-domain spectroscopy with the fit by Drude function, ${ }^{2}$ the accessible range of the carrier density was limited to a lower density than an order of $10^{16} \mathrm{~cm}^{-3}$, due to non-Drude behavior originating from nonthermal carrier distribution at the high carrier density region.

In doped semiconductors, such as $n$-type GaAs, it is well known that the plasmon and the longitudinal optical (LO) phonon form coupled modes through Coulomb interactions, and the frequencies of the LO phonon-plasmon coupled (LOPC) modes depend on the carrier density $N_{e}$ through the relation of the plasma frequency $\omega_{p}=\sqrt{4 \pi e^{2} N_{e} / \varepsilon_{\infty} m^{*}}$, where $\varepsilon_{\infty}$ is the high-frequency dielectric constant. ${ }^{3}$ Using Raman spectroscopy Nakashima and Harima ${ }^{4}$ have developed characterization of carrier mobility in SiC. They observed plasmonlike LOPC modes in frequency domain and fit the spectra with the line profile determined by frequency-dependent amplitude and dielectric function. As the results of the fitting, they obtained bandwidth of the plasmonlike LOPC mode, and put it into the formula $\mu=e /\left(m^{*} \gamma\right)$, where $\gamma$ is a damping rate of plasmon. ${ }^{4,5}$ The derived carrier mobility matched very well to that obtained by Hall measurements. Although Raman spectroscopy is a promising tool to estimate carrier mobility without any mechanical contact onto a sample, one need fitting of the line shape using a number of equations.

${ }^{a)}$ Electronic mail: mhase@bk.tsukuba.ac.jp.
Very recently, Huber et al. ${ }^{6}$ have revealed ultrafast build-up of the LOPC modes in InP by using optical pump-terahertz probe spectroscopy, however, relaxation dynamics of the LOPC modes and carrier mobility have not been examined.

In this letter, the direct measurement of carrier mobility in polar-semiconductor is proposed using a femtosecond optical pump-probe technique. Relaxation dynamics of strongly damped LOPC modes are observed in time-frequency space, which enables us to extract $\tau$. By using $\tau$, the electron mobility in $n$-type semiconductor is obtained at different photodoping levels, and found that the mobility is suppressed at high photodoping levels due to electron-hole scattering, while it is significantly enhanced near the critical carrier density.

To detect the plasmonlike LOPC modes in real time domain, we use an electro-optic detection ${ }^{7}$ combined with a standard pump-probe method utilizing a mode-locked Ti:sapphire laser with a pulse duration of $15 \mathrm{fs}$, and a center wavelength of $815 \mathrm{~nm}(=1.52 \mathrm{eV})$. This ultrashort pulse laser enables us to detect optical responses over $40 \mathrm{THz}$ bandwidth. The average power of pump-beam was varied from 10 to $200 \mathrm{~mW}$, while that of the probe was kept at $4 \mathrm{~mW}$. The maximum photoexcited carrier density by the pump light was estimated to be $N_{\text {exc }}=1.8 \times 10^{18} \mathrm{~cm}^{-3}$ from the pump power density and the absorption coefficient. The sample used was Si-doped $n$-type GaAs with a doped carrier density of $N_{\text {dop }}=1.0 \times 10^{18} \mathrm{~cm}^{-3}$. In our experimental condition, the total density of electron plasma is larger than that of hole plasma, and thus the phonon-plasmon coupling is dominated by photogenerated electron plasma rather than photogenerated hole plasma. In addition to this effect, the relaxation of the photogenerated holes is extremely fast $(\leq 75 \mathrm{fs}),{ }^{8}$ which would make hole plasma difficult to couple with the LO phonons. The transient anisotropic reflectivity change $\left(\Delta R_{\mathrm{eo}} / R\right)$ was recorded as a function of the time delay at room temperature.

The time derivatives of $\Delta R_{\mathrm{eo}} / R$ signal for $n$-GaAs at the two different photoexcited carrier densities are shown in Fig. 1. The coherent oscillations with a strong mode beating are clearly observed, which is due to the coexistence of the LO phonon $(8.75 \mathrm{THz})$, and the lower $\left(L_{-}\right)$and the upper $\left(L_{+}\right)$ branches of the LOPC modes. ${ }^{9,10}$ As shown in the Fourier 

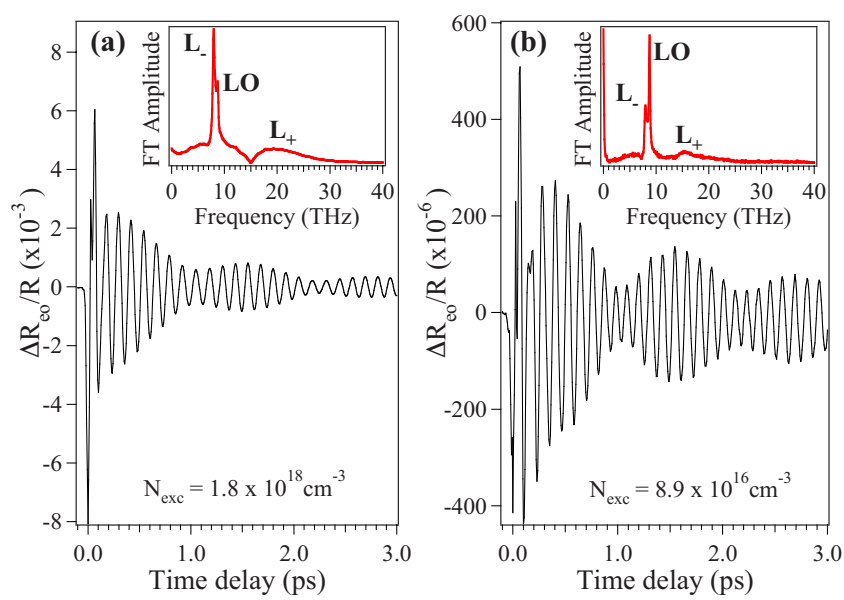

FIG. 1. (Color online) Time derivatives of the transient reflectivity changes for $n$-GaAs obtained by electro-optic detection at $300 \mathrm{~K}$. The inset shows their FT spectra.

transformed (FT) spectra in the inset of Fig. 1(a), the peak frequency of the $L_{-}$and the $L_{+}$modes at $N_{\text {exc }}=1.8$ $\times 10^{18} \mathrm{~cm}^{-3}$ are 7.9 and $19 \mathrm{THz}$, respectively. On the other hand, at the lowest limit of $N_{\mathrm{exc}}=8.9 \times 10^{16} \mathrm{~cm}^{-3}$ they are 7.9 and $15 \mathrm{THz}$, respectively, as shown in Fig. 1(b). ${ }^{10,11} \mathrm{Ob}-$ viously, the fit of the time-domain data using exponentially damped harmonic oscillations are difficult because of the complicated coherent oscillations appearing at the early time delay of $<300$ fs.

In order to explore the time-frequency dynamics of coupled plasmon-phonon system, a wavelet analysis ${ }^{12}$ was applied to the time-domain data in Fig. 1. Figure 2 shows three-dimensional image of electro-optic response obtained by the wavelet analysis. At the high photodoping level [Fig. 2 (a)], the transient frequency of the $L_{+}$mode $(23 \mathrm{THz})$ is higher than that observed in the static FT spectra $(19 \mathrm{THz})$, and it decays more quickly than those of the LO and the $L_{-}$ modes, which generate mode-beating patterns at $8-9 \mathrm{THz}$, living until a few picoseconds after photoexcitation. The higher frequency of the $L_{+}$mode $(23 \mathrm{THz})$ than that observed in the static FT in Fig. $1(19 \mathrm{THz})$ is possibly due to transient nonequilibrium electron distribution (hot electrons), ${ }^{1}$ which has much higher electron velocity than that of the cold
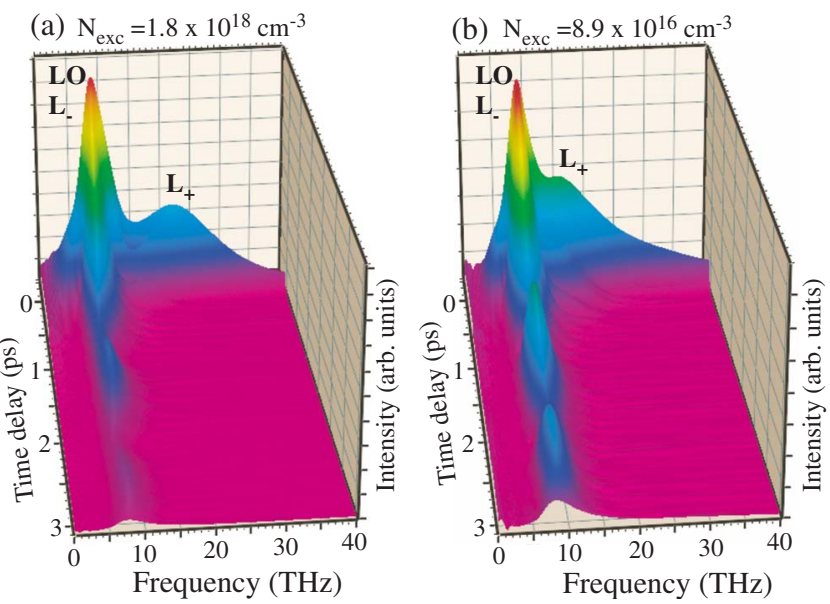

FIG. 2. (Color online) Three-dimensional images of the electro-optic response obtained by the wavelet analysis. (a) $N_{\mathrm{exc}}=1.8 \times 10^{18} \mathrm{~cm}^{-3}$ and (b) $N_{\text {exc }}=8.9 \times 10^{16} \mathrm{~cm}^{-3}$. The beating pattern observed at $\sim 8.7 \mathrm{THz}$ is due to the two modes of the LO $(8.75 \mathrm{THz})$ and the $L_{-}$mode $(7.9 \mathrm{THz})$.
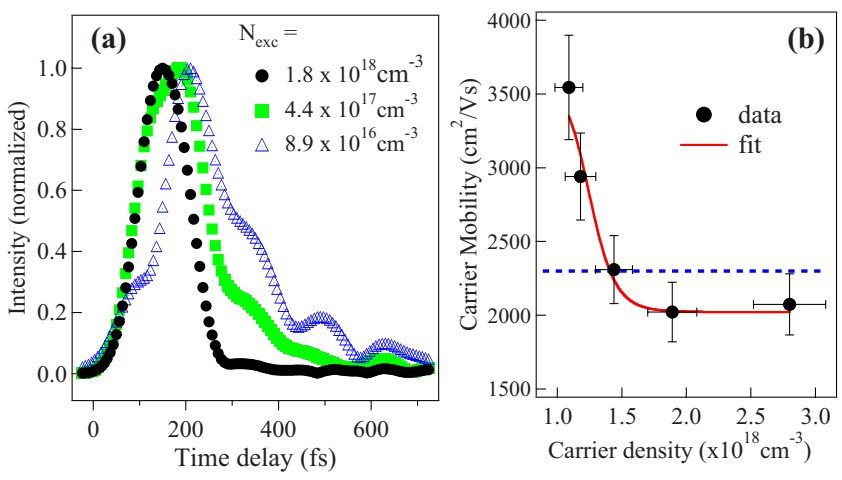

FIG. 3. (Color online) (a) Time evolution of the peak intensity of the plasmonlike LOPC mode at $23 \mathrm{THz}$ for $N_{\mathrm{exc}}=1.8 \times 10^{18} \mathrm{~cm}^{-3}, 20 \mathrm{THz}$ for $N_{\text {exc }}=4.4 \times 10^{17} \mathrm{~cm}^{-3}$, and $16 \mathrm{THz}$ for $N_{\text {exc }}=8.9 \times 10^{16} \mathrm{~cm}^{-3}$. The relaxation time $\tau$ is obtained by the decay curve of the peak intensity. (b) Carrier (electron) mobility extracted from the values of $\tau$ using $\mu=e \tau / m^{*}$ plotted as the function of the total carrier density. The horizontal dotted line at $2300 \mathrm{~cm}^{2} / \mathrm{V}$ s represents the Hall mobility of GaAs measured without photodoping.

plasma accommodating at the bottom of the conduction bands. $^{12}$ At the low photodoping level [Fig. 2(b)], on the other hand, the transient frequency of the $L_{+}$mode is only slightly higher $(16 \mathrm{THz})$ than that observed in the static FT spectra $(15 \mathrm{THz})$. In this lowest photodoping limit, the LOPC dynamics would be governed by the cold plasma, and thus the transient frequency of the $L_{+}$mode does not shift much compared to that observed in the static FT spectra. It is to be noted that the frequency of the $L_{+}$mode $(15 \mathrm{THz})$ matches to that observed by Raman measurement at the similar carrier doping level. ${ }^{13}$

In order to extract the relaxation time of the plasmonlike LOPC mode, the peak intensity of the mode is plotted as the function of the time delay at three different photodoping levels at the fixed majority carrier density of $N_{\text {dop }}=1.0$ $\times 10^{18} \mathrm{~cm}^{-3}$, as shown in Fig. 3(a). It is noticed that the rise time of the peak significantly depends on the photodoping levels. It decreases from $120 \pm 15$ to $73 \pm 15 \mathrm{fs}$ as the photodoping increases. This behavior is similar to the carrier density dependence of the buildup time of LOPC modes observed in photoexcited InP, and would be explained by the fastest corrective response time of the many-body system. ${ }^{6}$ The current interest is the relaxation time rather than the build-up time. The relaxation time $\tau$ of the plasmonlike LOPC mode also depends on the photodoping levels. The value of $\tau$ decreases from $135 \pm 15$ to $79 \pm 15$ fs as the photodoping increases, which is the similar effect observed in InN, ${ }^{14}$ and in the slightly low photodoped $n$-GaAs. ${ }^{10}$ Since the relaxation time of the plasmonlike LOPC mode can be directly corresponded to the carrier relaxation time $\tau$ under the condition that the dephasing of the plasmonlike LOPC mode $\left(L_{+}\right.$mode) is governed by the damping of the plasmon rather than that of the LO phonon, ${ }^{10,15}$ one can calculate the carrier mobility by $\mu=e \tau / m^{*}$. Here, we assume that there is no scattering of electrons into $X$ or $L$ valleys and the parabola conduction band structure at $\Gamma$ point, in which the standard value of the electron effective mass $\left(m^{*}=0.067 m_{e}\right)$ can be used. ${ }^{1}$

Figure 3(b) shows the carrier (electron) mobility extracted by using $\mu=e \tau / m^{*}$, where $e=1.602 \times 10^{-19} \mathrm{C}$ and $m_{e}=9.1 \times 10^{-31} \mathrm{~kg}$. The mobility exhibits monotonic decrease with increasing the carrier density with a saturation at 
the higher density than $N_{\text {tot }} \approx 1.8 \times 10^{18} \mathrm{~cm}^{-3}$, where $N_{\text {tot }}$ $=N_{\text {dop }}+N_{\text {exc }}$. Since we are changing the photodoping levels only, enhanced impurity scattering or temperature-dependent polar phonon (Fröhlich) scattering cannot account for the change in the mobility. Hence, the increased density of carriers plays a dominant role in that change. The mobility can be fit well to the empirical Caughey-Thomas ${ }^{16}$ relation in which all the scattering mechanisms are empirically included, ${ }^{17}$

$$
\mu=\frac{\mu_{\max }-\mu_{\text {min }}}{1+\left(N_{\text {tot }} / N_{\text {ref }}\right)^{\alpha}}+\mu_{\text {min }},
$$

where $N_{\text {ref }}$ and $\alpha$ denote fitting parameters, and $N_{\text {ref }}=1.26$ $\times 10^{18} \mathrm{~cm}^{-3}$ and $\alpha=13.2$ are obtained. Although the model describe the behavior only qualitatively, the relatively strong decrease in the mobility at lower density than $N_{\text {tot }} \approx 1.8$ $\times 10^{18} \mathrm{~cm}^{-3}$ would be dominated by electron-hole scattering, ${ }^{10,14}$ and the saturation of the mobility at higher density would come from the slow down of the carrier relaxation due to reabsorption of carriers by the high density hot phonons $^{2,18}$ or screening effect of Coulomb interactions. ${ }^{14}$ When we compare the mobility obtained in the present study with that by Hall measurement $\left(2300 \mathrm{~cm}^{2} / \mathrm{V} \mathrm{s}\right)$, it is found that the drift mobility $\mu$ determined by the coherent phonon spectroscopy is higher than the Hall mobility at $N_{\text {tot }} \leq 1.4$ $\times 10^{18} \mathrm{~cm}^{-3}$. Since the observed LOPC modes are always plasmonlike at $N_{\text {tot }} \geq 1.0 \times 10^{18} \mathrm{~cm}^{-3}$, ${ }^{10}$ transformation of the LOPC modes from plasmonlike into phononlike would play negligible role in the enhanced mobility at $N_{\text {tot }} \leq 1.4$ $\times 10^{18} \mathrm{~cm}^{-3}$. There are a few plausible explanations for the enhanced electron mobility: (i) the coherent coupling of LO phonon and the plasmon through Coulomb interactions ${ }^{19}$ and (ii) the difference between the two experimental methods. Regarding to the reason (ii) above, the Hall mobility $\mu_{H}$ can generally be expressed by $\mu_{H}=r_{H} \mu$, where $r_{H}$ is the Hall ratio (or Hall factor). ${ }^{1}$ The value of $r_{H}$ depends on the scattering mechanisms that contribute to the relaxation time $\tau$. In our case (Fig. 3), $r_{H}$ varies from $r_{H} \leq 1.0$ at $N_{\text {tot }} \leq 1.4$ $\times 10^{18} \mathrm{~cm}^{-3}$ to $r_{H} \approx 1.15$ at $N_{\text {tot }} \geq 1.8 \times 10^{18} \mathrm{~cm}^{-3}$. These facts signify that the scattering mechanisms contributing to $\tau$ in the present study is significantly different from that of the simple electron gas drifting under the weak and static electric field found in Hall measurements. The main scattering mechanism of nonequilibrium electron gas is governed by electron-hole scattering, ${ }^{10,20}$ while that of the simple (equilibrium) electron gas is affected by impurity and polar phonon (Fröhlich) scatterings as well as carrier-carrier scattering. Regarding to the reason (i) above, the enhanced carrier mobility observed by the coherent spectroscopy implies that one may manipulate the carrier mobility by controlling the Coulomb interaction via coherent control of the LOPC modes. ${ }^{21}$ It should be noted that the proposed method is valid only if the coupled mode are completely plasmonlike whose dephasing is determined by the plasmon damping.

In conclusion, the carrier (electron) mobility was determined from the dephasing time of the plasmonlike coherent LOPC mode $\left(L_{+}\right)$in $n$-GaAs, which is obtained by mapping the time-frequency dynamics of the LOPC modes by the use of the wavelet analysis. The electron mobility extracted from the coherent phonon spectroscopy decreases with increasing the photodoping levels, indicating the suppression of the mobility by enhanced electron-hole scattering. The availability of this technique will spread over the polar semiconductors, such as $\mathrm{SiC}$ and $\mathrm{GaN}$, under the condition that the photodoping level assures that the LOPC mode is plasmonlike.

This work was supported in part by Grant No. KAKENHI-19540329 from MEXT, Japan.

${ }^{1}$ P. Y. Yu and M. Cardona, Fundamentals of Semiconductors (Springer, Berlin, Heidelberg, 1999), Chap. 3.

${ }^{2}$ M. C. Beard, G. M. Turner, and C. A. Schmuttenmaer, Phys. Rev. B 62, 15764 (2000).

${ }^{3}$ A. Mooradian and A. L. McWhorter, Phys. Rev. Lett. 19, 849 (1967).

${ }^{4}$ S. Nakashima and H. Harima, Phys. Status Solidi A 162, 39 (1997).

${ }^{5}$ M. Chafai, A. Jaouhari, A. Torres, R. Antón, E. Martín, J. Jiménez, and W. C. Mitchel, J. Appl. Phys. 90, 5211 (2001).

${ }^{6}$ R. Huber, C. Kübler, S. Tübel, A. Leitenstorfer, Q. T. Vu, H. Hang, F. Köhler, and M.-C. Amann, Phys. Rev. Lett. 94, 027401 (2005).

${ }^{7}$ T. Dekorsy, G. C. Cho, and H. Kurz, in Light Scattering in Solids VIII, edited by M. Cardona and G. Güntherodt (Springer, Berlin, Heidelberg, 2000), Chap. 4.

${ }^{8}$ F. Ganikhanov, K. C. Burr, and C. L. Tang, Appl. Phys. Lett. 73, 64 (1998).

${ }^{9}$ G. C. Cho, T. Dekorsy, H. J. Bakker, R. Hövel, and H. Kurz, Phys. Rev. Lett. 77, 4062 (1996)

${ }^{10}$ M. Hase, S. Nakashima, K. Mizoguchi, H. Harima, and K. Sakai, Phys. Rev. B 60, 16526 (1999)

${ }^{11}$ The main difference between the previous experiment (Ref. 10) and the present experiment is the bandwidth of the detection $(<30 \mathrm{THz}$ in the previous one), which enabled us to observe plasmonlike LOPC modes at the higher total carrier density than those in the previous study.

${ }^{12}$ M. Hase, M. Kitajima, A. M. Constantinescu, and H. Petek, Nature (London) 426, 51 (2003).

${ }^{13}$ S. Nakashima, K. Mizoguchi, H. Harima, and K. Sakai, J. Lumin. 76-77, 6 (1998).

${ }^{14}$ Y.-M. Chang, H. W. Chu, C.-H. Shen, H.-Y. Chen, and S. Gwo, Appl. Phys. Lett. 90, 072111 (2007).

${ }^{15}$ F. Vallée, F. Ganikhanov, and F. Bogani, Phys. Rev. B 56, 13141 (1997).

${ }^{16}$ D. M. Caughey and R. E. Thomas, Proc. IEEE 55, 2192 (1967).

${ }^{17}$ The data in Fig. 3(b) was fit to also the power law of $\mu \sim N_{\text {tot }}^{-1}$ and $\mu$ $\sim N_{\text {tot }}^{-1 / 3}$, according to the Drude model and the Coulomb screening model, respectively (Ref. 14). The former model reproduced the reduction in $\mu$ at $N_{\text {tot }}<1.4 \times 10^{18} \mathrm{~cm}^{-3}$ well, while the latter one explained the behavior of $\mu$ at $N_{\text {tot }}>1.4 \times 10^{18} \mathrm{~cm}^{-3}$.

${ }^{18}$ H. P. M. Pellemans and P. C. M. Planken, Phys. Rev. B 57, R4222 (1998)

${ }^{19}$ J. D. Lee and M. Hase (unpublished).

${ }^{20}$ J. R. Meyer and F. J. Bartoli, Phys. Rev. B 28, 915 (1983).

${ }^{21}$ J. D. Lee and M. Hase, Phys. Rev. Lett. 101, 235501 (2008). 